\title{
Safety and Efficacy of Perampanel as Adjunctive Therapy in Patients with Refractory Focal Epilepsy Over 12 Months: Clinical Experience in a Real-World Setting
}

\author{
Fabrizio Rinaldi ${ }^{1}$ Giovanni De Maria ${ }^{1}$ \\ ${ }^{1}$ Epilepsy Unit, Spedali Civili di Brescia, University of Brescia, Pzz.le \\ Spedali Civili 1, 25100, Brescia, Italy
}

Int J Epilepsy 2018;5:75-79

\begin{abstract}
Address for correspondence Fabrizio Rinaldi, MD, Epilepsy Unit, Spedali Civili di Brescia, University of Brescia, Pzz.le Spedali Civili 1, 25100, Brescia, Italy (e-mail: rinaldi.fabrizio.riccardo@gmail.com).
\end{abstract}

\begin{abstract}
Background The main purpose of this study is to assess efficacy and tolerability of perampanel (PER), a noncompetitive $\alpha$-amino-3-hydroxy-5-methyl-4-isoxazole propionic acid receptor antagonist, as an add-on treatment in adult patients with refractory focal-onset seizures.

Patients and Methods A prospective, open label, observational study was conducted in patients with refractory focal-onset seizures treated with PER at our Epilepsy Unit, from May 2015 to February 2016. Patients were followed up for 1 year. Frequency of seizure and tolerability was assessed every 3 months. Patients were under a polytherapy, and the mean number of concomitant antiepileptic drugs (AEDs) at PER initiation was 2.9.

Results We consecutively enrolled 52 patients $(M / F=18 / 34)$. Three were lost on follow-up. Mean age was 38.7 years, with a mean duration of disease of 28.1 years. After 1 year of treatment, $57.14 \%$ reported a $50 \%$ or greater reduction in seizure frequency; five (10.21\%) were seizure free. Six (12.25\%) patients reported a reduction lower than 50\%. Mean dosage of PER was $7.57 \mathrm{mg}$. Thirty-one patients were taking enzyme-inducing AEDs (carbamazepine, oxcarbazepine, phenytoin). In this subgroup, the responder rate was $45.2 \%$. Twenty-one patients reported side-effects, most frequently somnolence (11), vertigo/ataxia (6), and aggressiveness (5). Eleven

Keywords

- antiepileptic drugs

- efficacy

- epilepsy

- perampanel

- real-world

- tolerability (22.4\%) patients reduced or discontinued at least one concomitant AED, while the electroencephalography improved in four (8.16\%). Sixteen (32.65\%) patients withdrew PER, after a mean duration of 163 days, the mean dosage being $6.4 \mathrm{mg}$ (range 4-12). Conclusions Adjunctive PER can achieve clinically meaningful improvement, or even seizure freedom, in almost two-thirds of patients suffering from refractory focal-onset epilepsies. It seems similarly safe and well-tolerated. Enzyme-inducing AEDs may limit the efficacy of PER.
\end{abstract}

\section{Introduction}

Perampanel (PER) is an orally active, novel, highly selective, noncompetitive $\alpha$-amino-3-hydroxy-5-methyl-4-isoxazole propionic acid (AMPA)-receptor antagonist. ${ }^{12}$ PER is approved

\section{received}

July 27,2018

accepted after revision

September 5, 2018

published online

January 07, 2019

10.1055/s-0038-1675546

ISSN 2213-6320. in the European Union and United States for the adjunctive treatment of primary generalized and focal seizures in patients with epilepsy aged more than 12 years.

Phase III trials of up to 19 weeks duration have shown Class I evidence that adjunctive PER is effective in improving

Copyright @2018 Indian Epilepsy

License terms 
seizure control, with acceptable tolerability. ${ }^{3-5}$ Furthermore, literature data suggests that the efficacy of PER can be influenced by the concomitant intake of enzyme-inducing antiepileptic drugs (AEDs) such as carbamazepine (CBZ), oxcarbazepine (OXC), and phenytoin (PHT) ${ }^{6,7}$

The results from regulatory studies are not always predictive of the outcomes in a real-world clinical setting, ${ }^{8}$ mainly because of the patients' selection strict criteria. ${ }^{9}$ To date there are only a few number of studies investigating the "real-life" experiences with PER in drug-resistant focal epilepsies. ${ }^{10-18}$ We report the results of an observational study performed at the Epilepsy Unit of the Spedali Civili di Brescia Hospital (Italy) on the effectiveness and tolerability of PER as an add-on treatment in adult patients suffering from severe focal epilepsy. The aim of this study is to assess efficacy and tolerability of PER, used as an add-on treatment in patients (aged $\geq 12$ years) with refractory partial-onset seizures, in the daily clinical practice setting.

\section{Patients and Methods}

We consecutively enrolled all the adult patients who started PER as an adjunctive treatment at the Epilepsy Unit of the Spedali Civili di Brescia Hospital from May 2015 to February 2016 ("enrolment period"). Patients were followed up for at least 1 year.

All patients suffered from drug-refractory focal epilepsy. Seizure type and seizure frequency were clinically assessed before the initiation of PER. In all patients, PER was titrated based on SmPC: treatment was started with $2 \mathrm{mg} /$ day at bedtime, and was uptitrated by $2 \mathrm{mg} /$ day every 2 to 4 weeks up to a maximum of $12 \mathrm{mg} /$ day. PER was uptitrated until occurrence of good seizure control or appearance of side-effects.

After initiation of PER, patients were typically seen in consultation every 3 to 4 months. Clinicians and patients usually documented seizure frequency providing a monthly average since their previous visit. Occurrence of adverse events (AEs) was reported, as well as withdrawal due to any cause. Clinicians also evaluated reduction in concomitant antiepileptic drugs (AEDs) and electroencephalography (EEG) improvement. If AEs were reported, the line of action was different between patients and could result in a dose reduction or PER withdrawal depending on the severity of AE and efficacy of treatment.

Effectiveness was evaluated comparing seizure frequency between baseline and the mean frequency in the last 3 months before the last outpatient visit. At every follow-up visit, an EEG was also performed. The MD was also allowed to reduce doses of other AEDs due to lack of necessity due to the response to PER. Patients were considered as "responders" if having a seizure frequency reduction in at least 50\%. Aggravation was defined as any increase in seizure frequency. The same analysis was performed dividing the cohort in two subgroups of patients taking enzyme-inducing AEDs (CBZ, OXC, PHT) and non-enzyme-inducing AEDs. In case of discontinuation, the reason was specified.

\section{Results}

\section{Patient Characteristics}

The PER was prescribed to 52 patients $(M / F=18 / 34)$ during the enrolment period. All patients were followed up for at least 1 year. Three patients ( 2 females) were lost on follow-up; the following analyses were then performed on the remaining 49 patients. The median age was 38.7 years (range 16-65), and 32 patients (65.3\%) were female. Thirty patients were affected by structural-metabolic epilepsy, while 19 patients by epilepsy of unknown etiology. Seizure types were focal in 38 patients, and focal and secondarily generalized in 11 patients. Mean duration of epileptic disease was 28.1 years; in seven (14.3\%) patients the onset of epilepsy was within 1 year of age. The mean number of concomitant AEDs at PER initiation was 2.9.

Three (6.1\%) patients had been previously submitted to epilepsy surgery, and three $(6.1 \%)$ patients had vagal nerve stimulator. Learning disability was present in 13 (26.5\%) patients and psychiatric comorbidity in 7 (14.3\%) patients. Mean seizure frequency at baseline was $13.4 /$ month (range 2-60); 10 (20.4\%) patients had daily seizures. Demographic and clinical details of patients are summarized in - Table $\mathbf{1}$

Table 1 Demographic and clinical details of 49 patients

\begin{tabular}{|c|c|}
\hline Women, $n(\%)$ & $32(65.3 \%)$ \\
\hline Mean age, years (SD) & $38.7(12.4)$ \\
\hline Duration of epilepsy, years (SD) & $28.1(12.6)$ \\
\hline \multicolumn{2}{|l|}{ Comorbidities, $n(\%)$} \\
\hline Intellectual and developmental disability & $13(26.5 \%)$ \\
\hline Psychiatric comorbidity & 7 (14.3\%) \\
\hline \multicolumn{2}{|l|}{ Epilepsy etiology, n (\%) } \\
\hline Structural/metabolic & $30(61.2 \%)$ \\
\hline Unknown & $18(36.7 \%)$ \\
\hline Other & $1(2.1 \%)$ \\
\hline \multicolumn{2}{|l|}{ Treatment } \\
\hline Surgery, $n(\%)$ & $3(6.1 \%)$ \\
\hline Vagus nerve stimulation, $n(\%)$ & $3(6.1 \%)$ \\
\hline \multicolumn{2}{|l|}{ Number of concomitant AEDs, $n$ (\%) } \\
\hline No AEDs & 0 \\
\hline $1 \mathrm{AED}$ & $2(4.1 \%)$ \\
\hline 2 AEDs & $17(34.7 \%)$ \\
\hline 3 AEDs & $18(36.7 \%)$ \\
\hline 4 AEDs & $9(18.4 \%)$ \\
\hline 5 or more & $3(6.12 \%)$ \\
\hline Mean number of concomitant AED & 2.9 \\
\hline \multicolumn{2}{|l|}{ Number of previous AEDs } \\
\hline Mean (SD) & $7.7(3.2)$ \\
\hline Range & $2-18$ \\
\hline Hepatic enzyme inducers, $n(\%)$ & $31(63.3 \%)$ \\
\hline
\end{tabular}

Abbreviations: AEDs, antiepileptic drugs; SD, standard deviation. 


\section{Efficacy}

After 12 months since PER initiation, mean PER dosage was 7.57 $\mathrm{mg} /$ day (standard deviation, SD 2.5, range $4-12 \mathrm{mg} /$ day). The responder rate was $57.14 \%$ (28 patients); five (10.2\%) patients were seizure free. In addition, six (12.2\%) patients reported a seizure reduction of less than 50\% (see - Table 2). Four (8.16\%) patients had an improvement in their EEG, and eleven (22.4\%) patients reduced the dosage of at least one concomitant AED. Seizure worsening was reported in five (10.2\%) patients.

Compared with the whole set of patients, the subgroup that reached seizure freedom had a later mean age-of-onset of disease (17.6 vs. 9.9 years), a shorter duration of epilepsy (21.6 vs. 28.6 years), and a lower monthly seizure frequency at baseline ( 2.8 vs. 14.6 ). Three patients achieved seizure freedom in the first 3 months after PER initiation, while the other two attained the same result after 6 and 9 months, respectively. They became seizure free with a mean dosage of $6.4 \mathrm{mg}$ of PER after 1 year of treatment.

When subdividing the patients based on the etiology, we found that the responder rate in patients affected by epilepsy of unknown etiology was higher than in structural-metabolic epilepsy, respectively, 68.4 versus $50.0 \%$.

\section{Adverse Effects}

Twenty-one (42.9\%) patients reported at least one AE. The most frequent were sedation in $11(22.4 \%)$ patients, dizziness/ataxia in $6(12.2 \%)$ patients, behavioral changes (mainly aggressivity) in $5(10.2 \%)$ patients, and headache in $3(6.1 \%)$ patients. Other complaints were gastrointestinal disturbances, weight gain, transient blurred vision, and falls ( - Table 3 ). Three out of five

Table 2 Effect on seizure frequency after 1 year of treatment with PER

\begin{tabular}{|l|l|l|}
\hline Nonresponders & $\boldsymbol{n}$ & $\%$ \\
\hline Worsened & 5 & 10.2 \\
\hline Unchanged & 10 & 20.4 \\
\hline Reduction $<50 \%$ & 6 & 12.2 \\
\hline Total & 21 & 43 \\
\hline Responders: & $n$ & $\%$ \\
\hline Reduction $>50 \%$ & 23 & 46.9 \\
\hline Seizure free & 5 & 10.2 \\
\hline Total & 28 & 57 \\
\hline
\end{tabular}

Abbreviation: PER, perampanel.
(60\%) patients that developed aggressive behavior had a history of learning disability, compared with 10 out of 44 patients (22\%) who did not display this adverse effect.

Sixteen (32.65\%) patients withdrew PER, after a mean duration of treatment of 163.1 days ( $S D=100.2$, range 28-336 days). In four (8.2\%) patients, this was due to lack of efficacy, while in $5(10.2 \%)$ patients, the reason for discontinuation was the presence of intolerable AEs. Mean dosage of PER in this subgroup of patients was $6.4 \mathrm{mg}$ (range: 4-12).

When comparing the patient who did develop any $\mathrm{AE}$ with the ones who did not, we found that the former was taking a lower dose of PER (6.6 mg vs. $7.5 \mathrm{mg}$ ), had a higher seizure frequency at baseline ( 15.9 vs. 11.5 ), and were taking the same number of comedication at baseline (2.95 vs. 2.82).

\section{Comedication with Hepatic Enzyme-Inducing Antiepileptic Drugs}

Thirty-one patients (63.3\%) were also taking hepatic enzyme-inducing AEDs (CBZ, OXC, and PHT). In this subgroup of patients, efficacy was lower than in patients not taking hepatic enzyme-inducing AEDs. These patients also displayed a higher frequency of AEs and a higher rate of withdrawal from medication. The data are summarized in - Table 4.

\section{Discussion}

The efficacy, safety, and effectiveness of PER as an add-on treatment in patients with focal and generalized seizures have been demonstrated in controlled trials. The effects of PER in the everyday clinical practice are still being studied, especially regarding the side-effects profile and the possible factors leading to a better response to treatment.

Table 3 Summary of adverse events

\begin{tabular}{|l|l|l|}
\hline & $n$ & $\%$ \\
\hline Sedation & 11 & 22.45 \\
\hline Dizziness/ataxia & 6 & 12.25 \\
\hline Aggressivity & 5 & 10.2 \\
\hline Headache & 3 & 6.12 \\
\hline Nausea & 2 & 4.08 \\
\hline Blurred vision & 1 & 2.04 \\
\hline Falls & 1 & 2.04 \\
\hline Weight gain & 1 & 2.04 \\
\hline
\end{tabular}

Table 4 Efficacy and side-effects in patients with and without enzyme-inducing AEDs

\begin{tabular}{|l|l|l|l|l|}
\hline & $\begin{array}{l}\text { Patients with enzymatic } \\
\text { inducer }(\boldsymbol{n}=31)\end{array}$ & $\begin{array}{l}\text { Patients without enzymatic } \\
\text { inducer }(\boldsymbol{n}=18)\end{array}$ & OR & $\boldsymbol{p}$-Value \\
\hline Seizure freedom, $\mathrm{n}(\%)$ & $1(3.2 \%)$ & $4(22.2 \%)$ & 0.11 & 0.05 \\
\hline$>50 \%$ seizure reduction, $\mathrm{n}(\%)$ & $14(45.2 \%)$ & $14(77.7 \%)$ & 0.23 & 0.03 \\
\hline Worsened seizure frequency, $\mathrm{n}(\%)$ & $5(16.1 \%)$ & $0(0 \%)$ & 0.43 & 0.14 \\
\hline Side-effects, $\mathrm{n}(\%)$ & $15(48.4 \%)$ & $6(33.3 \%)$ & 1.87 & 0.37 \\
\hline Withdrawal of medication, $\mathrm{n}(\%)$ & $13(41.9 \%)$ & $3(16.6 \%)$ & 3.61 & 0.11 \\
\hline
\end{tabular}

Abbreviations: AEDs, antiepileptic drugs; OR, odds ratio.

*Two-tailed Fisher's exact test. 
In our cohort of adult patients with focal refractory epilepsy treated with PER for 1 year, we observed a responder rate of $57.14 \%$, including $10.2 \%$ seizure-free patients. These results are consistent with the outcomes reported in the regulatory studies, ${ }^{10,11}$ and show a better efficacy compared with some of the real-world studies. ${ }^{12,19}$

One possibility for the differences between these studies could be a diverse epilepsy severity between groups. Nevertheless, our cohort of patients represents a highly refractory sample, as can be seen by a high number of concomitant AEDs, long story of epilepsy, and large proportion of patients with learning disability or psychiatric comorbidity. One other possibility that can explain this difference may lie in the higher mean PER dosage ( $7.57 \mathrm{mg} /$ day) of our sample compared with those reported in previous investigations $\left(5.8 \mathrm{mg} / \mathrm{day}^{9}\right)$, and more similar to the regulatory studies $\left(8.8 \mathrm{mg} /\right.$ day $^{10}$ and $7.7 \mathrm{mg} /$ day $\left.{ }^{11}\right)$. Therefore, we can suggest that in clinical practice, when treating patients with an incomplete response to low PER dosage, it might be appropriate to proceed to further increase in PER dosage to achieve clinical improvements. Caution about potential increase in side-effects, that are also dose dependent, must be used. Furthermore, the characteristics of the seizure-free patients in our cohort suggest that patients with a shorter duration of disease and an overall less severe disease at baseline may achieve better results even with lower dosages.

We also found a better response to treatment in patients with epilepsy of unknown etiology when compared with structural-metabolic etiology. This finding is interesting and could help shed some light on the baseline characteristics of patients who would most benefit from treatment with PER.

Talking about side-effects, we found that $42.9 \%$ of patients experienced AEs, which is lower than what reported in pooled analysis of the phase III studies (77\% in Steinhoff et $\mathrm{al}^{20}$ ) as well as in other clinical samples (52.0\% in Steinhoff et al, ${ }^{11} 67.4 \%$ in Shah et $\mathrm{al}^{21}$ ). This is of relevance, considering the prospective nature of the study and the mean dosage of PER used in our sample. The most commonly reported AEs were dizziness and somnolence, similar to those reported in the core randomized controlled trials (RCTs) of PER and the real-world studies. ${ }^{10,11}$ Also, the most frequently reported psychiatric AE was aggressivity (8.16\%), mirroring the results of previous studies, ${ }^{10-12}$ while another study reported a higher frequency of behavior/ mood disturbances $\left(18.1 \%{ }^{19}\right)$. One reason for these discrepancies may lie in the different methods for evaluating psychiatric disturbances: in fact, we did not use specific assessment scales for psychiatric disorders, and this could have led to an underestimation of these AEs. Also, different baseline patients' characteristics could explain this variability. In our cohort, we found a higher seizure frequency at baseline in those reporting $\mathrm{AE}$, but a lower dosage of PER. This may be explained by the fact that, in those not displaying side-effects, it was possible to reach higher dosages. On the other hand, a higher seizure frequency at baseline could be an independent risk factor; further studies with higher number of patients are needed to address this issue.

Alongside demonstrating real-world safety and efficacy, we also attempted to clarify whether the comedication with hepatic enzyme-inducing AEDs was associated with inferior clinical responses or augmented risk of AEs. Patients using concomitant enzyme-inducing AEDs had lower clinical responses to PER, with a responder rate of $45.2 \%$ (versus $77.7 \%$ in the subgroup without enzyme-inducing AEDs), along with a higher frequency of $\mathrm{AE}$. This has also been reported in RCT and observational studies. ${ }^{6}$ These results support the hypothesis that PER concentrations are lower in patients with enzyme-inducing AEDs than in those without, ${ }^{7}$ causing a lower clinical effect. ${ }^{13}$ Subsequently, clinicians should consider an increase in the dose of PER when combining it with a hepatic inducer. Nevertheless, the high frequency of AEs in this subgroup suggests that adverse effects are not dependent on the PER concentrations.

Our study has some limitations. We included a cohort of patients with refractory epilepsy of various types and etiology. No specific psychiatric assessments were performed at baseline to detect behavioral and psychiatric comorbidity, and data about the blood concentrations of PER were not available. Alongside, the long duration and prospective nature of this work in a "real-life" setting provide reliable real-world data, offer the potential for a fuller dataset, and can offer information on longer-term safety. . $^{94,15,22}$

\section{Conclusion}

Adjunctive therapy with PER for treating focal seizures in refractory patients can achieve clinically meaningful improvement in a high percentage of patients. Furthermore, this treatment was generally well tolerated. Comedication with enzyme-inducing AED can limit the efficacy and tolerability of PER treatment.

Conflict of Interest

None declared.

\section{References}

1 Rektor I. Perampanel, a novel, non-competitive, selective AMPA receptor antagonist as adjunctive therapy for treatment-resistant partial-onset seizures. Expert Opin Pharmacother 2013;14(2):225-235

2 Rogawski MA, Hanada T. Preclinical pharmacology of perampanel, a selective non-competitive AMPA receptor antagonist. Acta Neurol Scand Suppl 2013;197(197):19-24

3 French JA, Krauss GL, Biton V, et al. Adjunctive perampanel for refractory partial-onset seizures: randomized phase III study 304. Neurology 2012;79(6):589-596

4 French JA, Krauss GL, Steinhoff BJ, et al. Evaluation of adjunctive perampanel in patients with refractory partial-onset seizures: results of randomized global phase III study 305. Epilepsia 2013;54(1):117-125

5 French JA, Krauss GL, Wechsler RT, et al. Perampanel for tonic-clonic seizures in idiopathic generalized epilepsy A randomized trial. Neurology 2015;85(11):950-957

6 Villanueva V, Garcés M, López-González FJ, et al. Safety, efficacy and outcome-related factors of perampanel over 12 months in a real-world setting: The FYDATA study. Epilepsy Res 2016;126:201-210

7 Gidal BE, Laurenza A, Hussein Z, et al. Perampanel efficacy and tolerability with enzyme-inducing AEDs in patients with epilepsy. Neurology 2015;84(19):1972-1980 
8 Marson AG, Williamson PR. Interpreting regulatory trials in epilepsy. Curr Opin Neurol 2009;22(2):167-173

9 Trinka E, Steinhoff BJ, Nikanorova M, Brodie MJ. Perampanel for focal epilepsy: insights from early clinical experience. Acta Neurol Scand 2016;133(3):160-172

10 Steinhoff BJ, Bacher M, Bast T, et al. First clinical experiences with perampanel-the Kork experience in 74 patients. Epilepsia 2014a;55(Suppl 1):16-18

11 Steinhoff BJ, Hamer $H$, Trinka E, et al. A multicenter survey of clinical experiences with perampanel in real life in Germany and Austria. Epilepsy Res 2014b;108(5):986-988

12 Coyle H, Clough P, Cooper P, Mohanraj R. Clinical experience with perampanel: focus on psychiatric adverse effects. Epilepsy Behav 2014;41:193-196

13 Gidal BE, Ferry J, Majid O, Hussein Z. Concentration-effect relationships with perampanel in patients with pharmacoresistant partial-onset seizures. Epilepsia 2013;54(8):1490-1497

14 Maurousset A, Limousin N, Praline J, Biberon J, Corcia P, De Toffol B. Adjunctive perampanel in refractory epilepsy: experience at tertiary epilepsy care center in tours. Epilepsy Behav 2016;61:237-241

15 De Liso P, Vigevano $\mathrm{F}$, Specchio $\mathrm{N}$, et al. Effectiveness and tolerability of perampanel in children and adolescents with refractory epilepsies-an Italian observational multicenter study. Epilepsy Res 2016;127:93-100

16 Krauss GL, Serratosa JM, Villanueva V, et al. Randomized phase III study 306: adjunctive perampanel for refractory partial-onset seizures. Neurology 2012;78(18):1408-1415
17 Rektor I, Krauss GL, Bar M, et al. Perampanel Study 207: longterm open-label evaluation in patients with epilepsy. Acta Neurol Scand 2012;126(4):263-269

18 Krauss GL, Perucca E, Kwan P, et al. Final safety, tolerability, and seizure outcomes in patients with focal epilepsy treated with adjunctive perampanel for up to 4 years in an open-label extension of phase III randomized trials: Study 307. Epilepsia 2018;59(4):866-876

19 Juhl S, Rubboli G. Perampanel as add-on treatment in refractory focal epilepsy. The Dianalund experience. Acta Neurol Scand 2016;134(5):374-377

20 Steinhoff BJ, Ben-Menachem E, Ryvlin P, et al. Efficacy and safety of adjunctive perampanel for the treatment of refractory partial seizures: a pooled analysis of three phase III studies. Epilepsia 2013;54(8):1481-1489

21 Shah E, Reuber M, Goulding P, Flynn C, Delanty N, Kemp S. Clinical experience with adjunctive perampanel in adult patients with uncontrolled epilepsy: a UK and Ireland multicentre study. Seizure 2016;34:1-5

22 Singh K, Shah YD, Luciano D, Friedman D, Devinsky O, Kothare SV. Safety and efficacy of perampanel in children and adults with various epilepsy syndromes: a single-center postmarketing study. Epilepsy Behav 2016;61:41-45 